\title{
Assorting Isolation Methods with Three Options for Biodiesel Production from Waste Cooking Oil
}

\author{
Mr. Somesh M U \\ Research Scholar \\ Department of Civil Engineering \\ UVCE, Jnanabharathi Bangalore \\ Karnataka, India-560056
}

\author{
Dr. Usha N Murthy \\ Professor \& Former Chairman \\ Department of Civil Engineering \\ UVCE, Jnanabharathi Bangalore \\ Karnataka, India-560056
}

\begin{abstract}
Biodiesel creation is deserving of preceded with the study and streamlining of generation strategies because of its earth advantageous qualities and its inexhaustible nature. From a waste-administration viewpoint, the creation of biodiesel from utilized cooking oil is ecologically useful since it gives a cleaner method for discarding these items than is ordinarily the situation. Biodiesel delivered by base-catalyzed transesterification of vegetable oil is generally performed in clump reactors where the required vitality is given by warming joined by mechanical blending. Utilizing this strategy, the best yield rate was acquired utilizing a methanol/oil molar proportion of 6:1, potassium hydroxide $(1 \%)$, and $65^{\circ} \mathrm{C}$ temperature for 60 minutes. On the other hand, ultra-sonication can give a viable method to accomplish the required blending while at the same time giving the important enactment vitality. It was presumed that trans-esterification by low recurrence ultrasound $(20 \mathrm{kHz})$ offered a lot of favorable circumstances over the ordinary established technique. It turned out to be effective (biodiesel yield

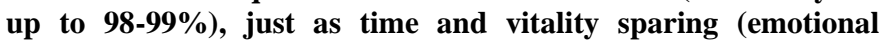
decrease of response time to $5 \mathrm{~min}$, contrasted with one hour or additionally utilizing ordinary clump reactor frameworks, and a surprising decrease in static detachment time to $25 \mathrm{~min}$, contrasted with 8 hours). The third alternative examined was the utilization of microwave light. The use of radio recurrence microwave vitality offered a quick, simple course to this important biofuel with favorable circumstances of upgrading the response rate and improving the partition procedure. The approach took into consideration the utilization of high FFA content feedstock, including utilized cooking oil, subsequently diminishing the expense of creation, which establishes a noteworthy obstacle towards broad commercialization of biodiesel.
\end{abstract}

Keywords:- Biodiesel, trans-esterification, enhancement, ultrasonication, microwave.

\section{INTRODUCTION}

The three fundamental techniques for ester generation from oils/fats are base-catalyzed transesterification, acid-catalyzed esterification, and enzymatic catalysis. The most regularly utilized strategy for which is the base-catalyzed transesterification system as it is the most affordable process[1]. Transesterification is a harmony

response and the change happens basically by blending the reactants. Notwithstanding, the nearness of an impetus quickens impressively the change of harmony [2]. Methanolysis with $1 \%$ wt. potassium hydroxide impetus brought about effective change giving the best yields and viscosities of the esters [3].
The stoichiometric response requires $1 \mathrm{~mol}$ of triglyceride and $3 \mathrm{~mol}$ of the liquor. Be that as it may, an overabundance of the liquor is utilized to build the yields of the alkyl esters and to permit its stage partition from the glycerol shaped [4]. The ideal methanol/oil molar proportion was observed to be $6: 1$ [3]. While transesterification is entrenched, and ending up progressively significant, there stay impressive wasteful aspects in existing transesterification forms. In traditional warming of transesterification process (a bunch, consistent, and too basic methanol process), heat vitality is exchanged to the crude material through convection, conduction, and radiation from surfaces of the crude material. Along these lines, the regular warming expends more vitality and take long preheat and response time, ideally 1 hour [3], to deliver more than 95 percent transformation yield biodiesel item.

Since this response can just happen in the interfacial district between the fluids and furthermore because of the way that fats and alcohols are not absolutely miscible transesterification is a generally moderate procedure. Subsequently, an incredible blending is required to expand the territory of contact between the two immiscible stages, and in this manner to create an emulsion [5]. Past research has shown that the ultrasonication gives the mechanical vitality to blending and the required vitality for starting the transesterification response [6-10]. Low recurrence ultrasonic light is a helpful instrument for emulsification of immiscible fluids. The breakdown of the cavitation bubbles upsets the stage limit and causes emulsification by ultrasonic planes that encroach one fluid to another [12].

An elective vitality stimulant, "microwave light" can be utilized for the creation of the elective vitality source, biodiesel. In the electromagnetic radiation range, the microwave radiation area is situated between infrared radiation and radio waves. Microwaves (MW) have wavelengths of $1 \mathrm{~mm}$ - 1m, relating to frequencies between 0.3 - $300 \mathrm{GHz}$. When all is said in done, so as to maintain a strategic distance from impedance, mechanical and household microwave contraption are managed to $12.2 \mathrm{~cm}$, comparing to a recurrence of $2.45 \mathrm{GHz}$, yet other recurrence allotments do exist [13].

\section{METHODOLOGY}

The tests have been kept running in triplicate; each arrangement of activity conditions was led multiple times, once with sunflower oil utilized as a kind of perspective (NVO) and twice with waste vegetable oils (WVO) got from 
two distinct sources: one gathered from a house (house vegetable oil $-\mathrm{WVOH}$ ) and the other from an inexpensive food famous café (Restaurant vegetable oil - WVOR). Methanol was the liquor of decision: Methanol (Analytical) Pharmaceutical Chemicals. Both $\mathrm{KOH}$ and $\mathrm{NaOH}$ were utilized in that review: $\mathrm{NaOH}$ pellets \& $\mathrm{KOH}$ pellets were filtered. The technique connected in that review was the soluble base-catalyzed transesterification. The transesterification procedure was learned at two impetus loadings $(0.5 \%$ and $1.0 \% \mathrm{KOH}$ wt./wt. $)$, two response temperatures $\left(25\right.$ and $65{ }^{\circ} \mathrm{C}$ ) and three liquor to-oil molar proportions (3:1, 6:1 and 9:1). Essentially, methanol was the liquor of decision and $\mathrm{KOH}$ was utilized as the impetus. The creation approach pursued was gotten by inquiring about current techniques for little scale generation. Potassium or sodium methoxide arrangement was arranged crisply by blending a foreordained measure of methanol (20\% by weight of oil) with $\mathrm{KOH}$ or $\mathrm{NaOH}(1.0 \%$ by weight of oil) in a holder. The response was completed for 1-3 h. under reflux at $60-70{ }^{\circ} \mathrm{C}$. Mixing was begun with the response right now of including potassium or sodium methoxide arrangement. After the foreordained response time, the blend was deliberately exchanged to an isolating channel and permitted to remain there medium-term. The lower layer (glycerol, methanol and a large portion of the impetuses) was depleted out. The upper layer (methyl esters, some methanol, and hints of the impetus) was then cleaned altogether by washing with warm $\left(50^{\circ} \mathrm{C}\right)$ de-ionized water. The methyl ester was then dried with anhydrous $\mathrm{Na} 2 \mathrm{SO} 4$.

The ideal parametric conditions acquired from the ordinary procedure were connected again utilizing ultrasonication and furthermore microwave light so as to look at the three frameworks. Ultrasonic blending was performed with an ultrasonic processor (VC 100, Sonics and Materials Inc.,). This processor utilizes electric excitation to create ultrasound, which is transmitted into the fluid blend by means of a titanium test that causes blending and gives the vitality to the transesterification. The processor works at 100 watts, $20 \mathrm{kHz}$ recurrence. The sufficiency and the beat for the response can be balanced from $0-100 \%$. Procedure parameters, for example, adequacy, beat, working time were regulated utilizing the control handles.

In this investigation, a logical microwave with cutting edge vessel innovation was utilized. This permitted quick vessel warming with homogeneous microwave dispersion all through the pit. The stove utilized is Start S (Milestone) Milestone Inc... The typical weight glass reactor is finished with a 500 $\mathrm{ml}$ carafe and reflux condenser. The stove is provided with a shading contact screen controller that empowers creation, stockpiling and utilization of time-versus. - Temperature or time-versus. - control response profiles. The yield microwave control is movable up to1200 watts, controlled by means of a chip. The temperature was changed in accordance with $65{ }^{\circ} \mathrm{C}$, a methanol/oil molar proportion of 6:1 was utilized, and potassium hydroxide $(1 \%)$ was utilized as an impetus. The oil was preheated to the ideal temperature of $65{ }^{\circ} \mathrm{C}$ utilizing the microwave unit. The alcohol- impetus blend was then sustained into the carafe through the condenser. The yield control was acclimated to 500 watts and the blend was lighted under reflux applying distinctive response times of 30,60, 90, 120, 150, 180 and 240 seconds.

\section{RESULTS\& DISCUSSION}

From the outcomes, obviously, the created biodiesel fuel was inside the suggested gauges of the biodiesel fuel with $96.15 \%$ yield. Biodiesel delivered from waste vegetable oil was tantamount in structure, comparable in calorific incentive to biodiesel created from refined sunflower oil.

An examination held between the response time, static detachment time and yield\% on utilizing ultrasonic blending versus mechanical mixing appear table 1.

The response time was diminished significantly from 1 hour to 5 minutes (92\%), the static division time was decreased amazingly from 8 hours to 25 minutes (95\%) and the yield\% was commonly higher.

Table 1: Comparison between mechanical and ultrasonic mixing at optimum conditions.

\begin{tabular}{|c|c|c|c|c|c|c|}
\hline & \multicolumn{2}{|c|}{ Yield\% } & \multicolumn{2}{c|}{ Reaction time (min) } & \multicolumn{2}{c|}{ Separation time (min) } \\
\hline Oil & Mechanical & Ultrasonic & Mechanical & Ultrasonic & Mechanical & Ultrasonic \\
& Mixing & Mixing & Mixing & Mixing & Mixing & Mixing \\
\hline NVO & 96.15 & 98.94 & 60 & 5 & 480 & 25 \\
WVOH & 95.79 & 98.26 & 60 & 5 & 480 & 25 \\
WVOR & 94.51 & 97.68 & 60 & 5 & 480 & 25 \\
& & & & & & \\
\hline
\end{tabular}

Table 2: Comparison between microwave irradiation and the conventional technique.

\begin{tabular}{|c|c|c|c|}
\hline System & $\begin{array}{c}\text { Reaction Time } \\
(\mathrm{min})\end{array}$ & $\begin{array}{c}\text { Separation time } \\
(\mathrm{min})\end{array}$ & Yield\% \\
\hline Conventional & 60 & 480 & 96 \\
\hline Microwave & 2 & 25 & 100 \\
\hline
\end{tabular}

Figure 1: Comparison between the reaction times of the three techniques.

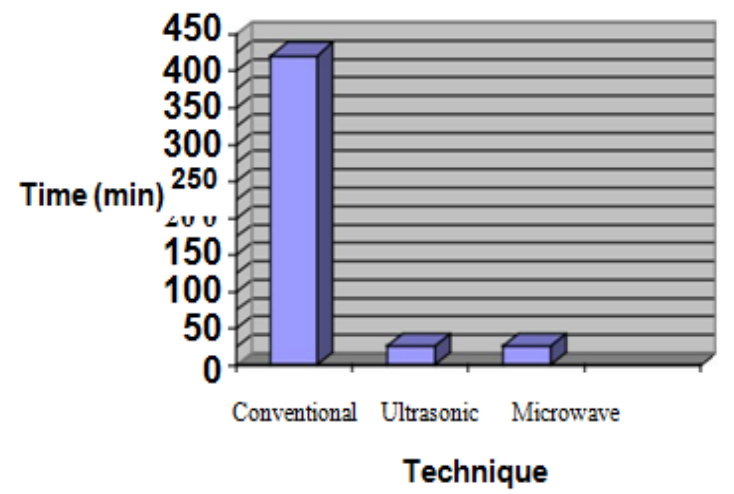


Figure 2: Comparison between the separation times of the three techniques.

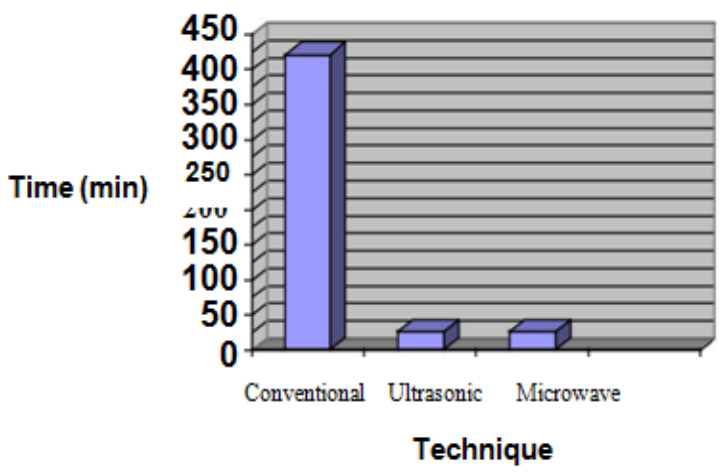

Figure 1 delineates a correlation between the response times utilizing the ordinary method, ultra-sonication and microwave illumination. In figure 2 a similar examination is held with respect to the division times.

\section{CONCLUSION AND RECOMMENDATIONS}

From the present examination, it very well may be presumed that low recurrence ultrasound is an effective, efficient and financially useful, offering plenty of points of interest over the traditional established system. The response time was decreased significantly from $1 \mathrm{hr}$ to $5 \mathrm{~min}(92 \%)$, the static detachment time was diminished surprisingly from $8 \mathrm{hr}$ to 25 minutes $(95 \%)$ and the yield\% was commonly higher. At a $20 \mathrm{kHz}$ recurrence, the ideal response time was observed to be 5 minutes and the ideal sufficiency was observed to be $100 \%$. Biodiesel that met the ASTM D 6751 standard was gotten with yields more than $98-99 \%$ at a detachment time of just 25 minutes.

The outcomes demonstrated that the utilization of radio recurrence microwave vitality offers a quick, simple course to this profitable biofuel with points of interest of upgrading the response rate and improving the division procedure. The strategy considers the utilization of high FFA content feedstock, including utilized cooking oil; subsequently, it decreases the expense of creation, which comprises a noteworthy obstacle towards broad commercialization of biodiesel.

\section{REFERENCES}

[1] Singh, A. B. He, Thompson, J. \& Van Gerpen, J., Process Optimization of Biodiesel Production using Different Alkaline Catalysts. Applied Engineering in Agriculture, 22(4), pp. 597-600, 2006.

[2] Ma, F. \& Hanna, M.A., Biodiesel Production: A Review. Bio Technology, 70, pp. 1-15, 1999

[3] Refaat, A.A., Attia, N.K., Sibak, H.A., El Sheltawy, S.T. \&ElDiwani, G.I., Production Optimization and Quality Assessment of Biodiesel from Waste Vegetable Oil. International Journal of Environmental Science and Technology, 5(1), pp. 75-82, 2008.

[4] Schuchardt, U., Serchelia, R. \& Vargas, R.M., Transesterification of Vegetable Oils: a Review. Journal of Brazilian Chemical Society, 9(1), pp. 199-210, 1998.

[5] Singh, A.K. \& Fernando S.D., Catalyzed Fast-Transesterification of Soybean Oil Using Ultrasonication. American Society of Agricultural Engineers, ASAE Annual Meeting, Paper number 066220, 2006.

[6] Kelkar, M.A., Gogate, P.R. \&Pandit, A.B. Intensification of esterification of acids for synthesis of biodiesel using acoustic and hydrodynamic cavitation. UltrasonicsSonochemistry, 15, pp. 188-194, 2008.

[7] Stavarache, C., Vinatoru, M. \& Maeda, Y., Aspects of ultrasonically assisted transesterification of various vegetable oils with methanol UltrasonicsSonochemistry, 14(3), pp. 380-386, 2007.

[8] Siatis, N.G., Kimbaris, A.C., Pappas, C.S., Tarantilis, P.A. \&Polissiou, M.G., Improvement of Biodiesel Production Based on the Application of Ultrasound: Monitoring of the Procedure by FTIR Spectroscopy Journal of the American Oil Chemists Society, 83(1), pp. 53-57, 2006.

[9] Hanh, H.D., Dong, N.T., Starvarache, C., Okitsu, K., Maeda, Y. \& Nishimura, R., Methanolysis of triolein by low frequency ultrasonic irradiation. Energy Conversion and Management, 49, pp. 276-280, 2008 .

[10] Stavarache, C., Vinatoru, M., Nishimura, R. \& Maeda, Y., Fatty acids methyl esters from vegetable oil by means of ultrasonic energy. UltrasonicsSonochemistry, 12, pp. 367-372, 2005.

[11] Colucci, J.A., Borrero, E.E. \&Alape, F., Biodiesel from an Alkaline Transesterification Reaction of Soybean Oil Using Ultrasonic Mixing. Journal of the American Oil Chemists Society, 82(7), pp. 525-530, 2005 .

[12] Stavarache, C., Vinatoru, M. \& Maeda, Y., Ultrasonic versus silent methylation of vegetable oils. UltrasonicsSonochemistry, 13(5), pp. 401-407, 2006.

[13] Lidstrom, P., Tierney, J., Wathey, B. \&Westman, J., Microwav assisted organic synthesis - a review. Tetrahedron, 57, pp. 9225-9283, 2001.

[14] Varma, R.S., Solvent-free accelerated organic syntheses using microwaves. Pure and Applied Chemistry, 73(1), pp. 193-198, 2001. 